\title{
Amount of time spent in sedentary behaviors and cause-specific mortality in US adults ${ }^{1-3}$
}

\author{
Charles E Matthews, Stephanie M George, Steven C Moore, Heather R Bowles, Aaron Blair, Yikyung Park, \\ Richard P Troiano, Albert Hollenbeck, and Arthur Schatzkin
}

\begin{abstract}
Background: Sedentary behaviors predominate modern life, yet we do not fully understand the adverse effects of these behaviors on mortality after considering the benefits of moderate-vigorous physical activity (MVPA).

Objective: We tested the hypotheses that higher amounts of overall sitting time and television viewing are positively associated with mortality and described the independent and combined effects of these sedentary behaviors and MVPA on mortality.

Design: In the NIH-AARP Diet and Health Study, we examined 240,819 adults (aged 50-71 y) who did not report any cancer, cardiovascular disease, or respiratory disease at baseline. Mortality was ascertained over $8.5 \mathrm{y}$.
\end{abstract}

Results: Sedentary behaviors were positively associated with mortality after adjustment for age, sex, education, smoking, diet, race, and MVPA. Participants who reported the most television viewing ( $\geq 7 \mathrm{~h}$ compared with $<1 \mathrm{~h} / \mathrm{d}$ ) were at greater risk of all-cause (HR: 1.61; 95\% CI: $1.47,1.76$ ), cardiovascular (HR: 1.85 ; 95\% CI: 1.56 , 2.20 ), and cancer (HR: 1.22 ; 95\% CI: $1.06,1.40$ ) mortality after adjustment for MVPA. Overall sitting was associated with all-cause mortality. Even among adults reporting high levels of MVPA ( $>7 \mathrm{~h} / \mathrm{wk})$, high amounts of television viewing $(\geq 7 \mathrm{~h} / \mathrm{d})$ remained associated with increased risk of all-cause (HR: $1.47 ; 95 \% \mathrm{CI}: 1.20,1.79)$ and cardiovascular (HR: 2.00; 95\% CI: 1.33, 3.00) mortality compared with those reporting the least television viewing $(<1 \mathrm{~h} / \mathrm{d})$.

Conclusions: Time spent in sedentary behaviors was positively associated with mortality, and participation in high levels of MVPA did not fully mitigate health risks associated with prolonged time watching television. Adults should be encouraged to reduce time spent in sedentary behaviors, when possible, and to participate in MVPA at recommended levels. The NIH-AARP Diet and Health Study was registered at clinicaltrials.gov as NCT00340015. Am J Clin Nutr 2012;95:437-45.

\section{INTRODUCTION}

Social and technological changes in the United States since the 1950s have substantially reduced overall physical activity levels and increased time spent in sedentary behaviors (1). Epidemiologic investigation into the health effects of a "sedentary lifestyle" has customarily focused on the adverse effects associated with a lack of participation in recommended levels of exercise, or moderate-vigorous physical activity (MVPA) (2, 3). However, our understanding of the potential adverse effects of time spent in sedentary behaviors on overall physical activity levels is evolving rapidly as the role of daily activities and nonexercise energy expenditure in health is better defined (3). Time spent in sedentary behaviors reflects a wide range of human pursuits that involve sitting or reclining and only low levels of energy expenditure (3). The average US adult spends more than half of his or her waking day in sedentary behaviors, and older adults spend upward of $60 \%$, or $\geq 9 \mathrm{~h}$, of their time each day in sedentary behaviors (4).

Recent studies report that higher amounts of sedentary time are independently associated with increased risk of weight gain and obesity $(5,6)$, poor metabolic health (7-9), and mortality (1012). Patel et al (11) reported that sitting during leisure time was positively associated with mortality even after overall physical activity levels were controlled for, and that high levels of total activity did not minimize risk related to sitting. Two studies reported similar findings on the independent and combined effects of activity and overall sitting time (10) and television viewing (12). However, none of these studies attempted to determine the amount of MVPA that may be required to mitigate the adverse effects of sedentary behaviors on mortality.

Accordingly, here we test the hypotheses that higher amounts of overall sitting time and more time watching television are positively associated with mortality from all causes, cardiovascular disease, and all cancers. To gain insight into the level of MVPA that is required to minimize health risks associated with high amounts of sedentary behavior we also describe the independent and combined effects of these 2 health behaviors on mortality. We hypothesize that time spent in sedentary behaviors displaces time spent in lower intensity nonexercise behaviors and results in a reduction in overall physical activity levels.

\footnotetext{
${ }^{1}$ From the Nutritional Epidemiology Branch (CEM, SMG, SCM, YP, and AS) and the Occupational Epidemiology Branch (AB), Division of Cancer Epidemiology and Genetics, and the Risk Factor Monitoring and Methods Branch, Division of Cancer Control and Population Sciences (HRB and RPT), National Cancer Institute, Rockville, MD; and the AARP, Washington, DC (AH).

${ }^{2}$ Supported in part by the Intramural Research Program of the NIH, National Cancer Institute.

${ }^{3}$ Address correspondence to CE Matthews, Nutritional Epidemiology Branch, Division of Cancer Epidemiology and Genetics, National Cancer Institute, 6120 Executive Boulevard, EPS 3028, Bethesda, MD 20892-7335. E-mail: charles.matthews2@nih.gov.

Received May 9, 2011. Accepted for publication November 1, 2011.

First published online January 4, 2012; doi: 10.3945/ajen.111.019620.
} 


\section{SUBJECTS AND METHODS}

The NIH-AARP Diet and Health Study was established in 1995-96 when 566,401 AARP members aged 50-71 y living in California, Florida, Louisiana, New Jersey, North Carolina, Pennsylvania, Atlanta, or Detroit responded to a questionnaire about their medical history, diet, and demographic characteristics (13). Within 6 mo after completing this survey, respondents who did not report colon, breast, or prostate cancer initially were asked to complete a second exposure assessment questionnaire that asked about sedentary behaviors and MVPA.

Eligible participants for the present study were those who responded to both questionnaires and who were alive and had not moved from the study area before returning the second questionnaire $(n=334,908)$. We excluded cohort members who reported a prior diagnosis of heart disease $(n=42,062)$, cancers other than basal cell skin cancer $(n=18,854)$, stroke $(n=3691)$, and emphysema $(n=5710)$ or those who rated their health status to be poor $(n=1467)$. We also excluded those who indicated that they were proxies for the intended respondents $(n=10,383)$ and participants with missing information on sedentary $(n=1964)$ and MVPA $(n=3106)$ behaviors, BMI values (in $\mathrm{kg} / \mathrm{m}^{2}$ ) that were extreme $(<15$ or $>60)$ or missing $(n=5327)$, and extreme log-transformed energy intake values $(n=1519)$. Our analytic cohort consisted of 240,819 participants. For cause-specific mortality analyses, we further excluded individuals with unknown causes of death $(n=281)$. The Special Studies Institutional Review Board of the US National Cancer Institute approved the study. Questionnaire completion was considered to imply informed consent.

\section{Cohort follow-up and endpoint ascertainment}

Cohort members were followed by linkage to the US Postal Service National Change of Address database, through processing of undeliverable mail, address change services, and direct contact with participants. Vital status was determined through linkage with the Social Security Administration Death Master File (14), and determinations of vital status and causes of death were made by using the National Death Index (15). The primary endpoints for our analysis were mortality from all causes, cardiovascular disease, and cancer.

\section{Assessment of sedentary behaviors and MVPA}

The following 3 distinct exposures were assessed: television viewing, overall sitting, and MVPA. Television viewing was assessed by the question, "During a typical 24-hour period over the past 12 months, how much time did you spend watching television or videos? (None, $<1,1-2,3-4,5-6,7-8$, or $9+\mathrm{h} / \mathrm{d}$ )." To assess overall sitting, participants were asked, "During a typical 24-hour period over the past 12 months, how much time did you spend sitting? $(<3,3-4,5-6,7-8$, or $9+\mathrm{h} / \mathrm{d})$." MVPA during leisure time was assessed as the average time spent each week in activities of at least a moderate intensity in the past 10 y (never, rarely, weekly but $<1 \mathrm{~h} /$ wk; $1-3 \mathrm{~h} /$ wk; 4-7 $\mathrm{h} / \mathrm{wk} ;>7 \mathrm{~h} / \mathrm{wk}$ ). Validity of these questions has not been evaluated, but they are similar to those used in assessments of sedentary behavior (16) and recreational moderate-vigorous activity (17), which have been noted to have acceptable validity; the question on MVPA has been associated with mortality (18).

\section{Covariate assessment}

A broad range of covariates was evaluated as potential confounders to the associations of interest (Table 1). Diet was measured by using a 124-item food-frequency questionnaire, and the Healthy Eating Index-2005 (HEI-2005) was used as a measure of overall diet quality (19).

\section{Statistical analysis}

Cox proportional hazards regression was used to estimate HRs and $95 \%$ CIs adjusted for age and relevant covariates. Follow-up time was calculated from the scan date on the second questionnaire until death from any cause or the end of the follow-up, 31 December 2005. Tests for trend were performed by entering the median values of each category in the models. Spearman correlations were used to describe the relation between exposures. Covariate selection for our models was developed with the following considerations. Covariates that acted as confounders (ie, changed the magnitude of associations by $\geq 10 \%$ ) with allcause mortality were retained in adjusted models for all of the mortality outcomes. Although race did not meet this definition of a confounder, it was retained in the model on an a priori basis because of known differences in TV viewing and rates of mortality by race. We present results from 3 models: 1) adjusted for age and sex; 2) adjusted for age (y), sex (male or female), race (white, black, other, or missing), education $(<12 \mathrm{y}$, high school graduate, some college, college graduate, or missing), smoking history (never; quit, $\leq 20$ cigarettes/d; quit, $>20$ cigarettes/d; current, $\leq 20$ cigarettes/d; current, $>20$ cigarettes/d; or unknown), and diet quality (quintiles) (model A); and 3) adjusted for covariates as for model A plus MVPA (never or rarely; $<1,1-3,4-7,>7 \mathrm{~h} / \mathrm{wk}$ ) (model B). We did not adjust for BMI in our primary models because sedentary behaviors are a cause of weight gain (20), and it is not always appropriate to adjust for causal intermediates in statistical models estimating disease risks (21). We report separately the effect on our results of adjustment for BMI reported at baseline in secondary analyses, with results presented in Supplementary Table 1 under "Supplemental data" in the online issue.

To describe the independent and combined effects of sedentary time and MVPA on mortality, we examined risk of these distinct exposures relative to a common low-risk referent group (ie, low sedentary behavior and high MVPA), after adjustment for model A covariates. We also examined multiplicative interactions for MVPA and television viewing by adding their cross-product to the statistical models. Stratified analysis was conducted to evaluate effect modification by selected factors, and sensitivity analysis evaluated our primary results after the exclusion of deaths in the first 3 y of follow-up. SAS 9.1 (SAS Institute Inc) was used, and statistical tests were 2-sided.

\section{RESULTS}

During $8.5 \pm 1.7$ y (mean $\pm \mathrm{SD}$ ) of follow-up, we ascertained 17,044 deaths from all causes, 4684 from cardiovascular disease, and 7652 from cancer. Sedentary behaviors were associated with age, nonwhite race, education, obesity, smoking, and level of MVPA, as well as a number of health conditions (hypertension, high cholesterol, diabetes, gall bladder disease, and bone health) and dietary quality (Table 1). 


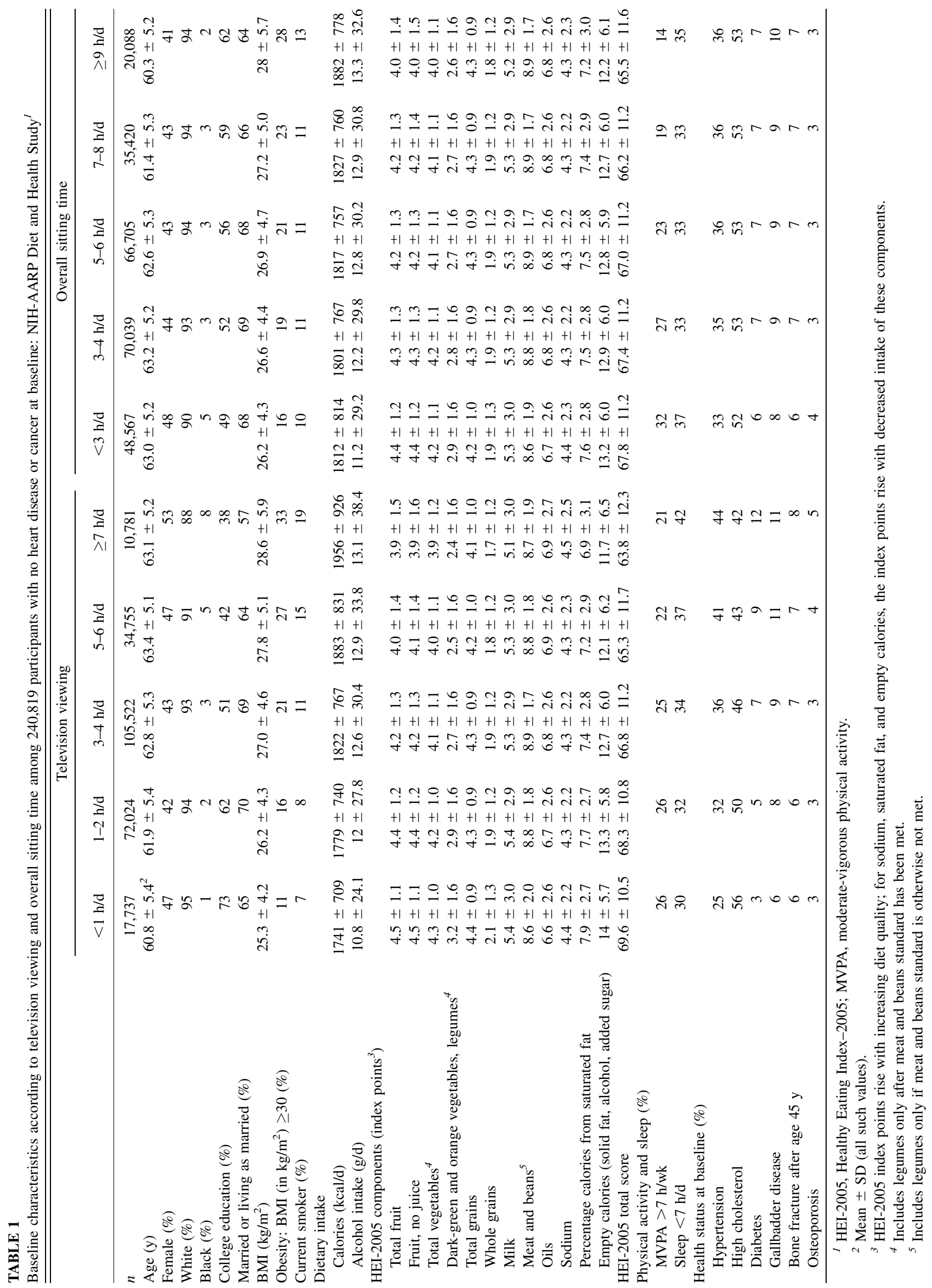


The correlation between overall sitting and television time was weak $(r=0.20, P<0.01)$ as were correlations between MVPA and overall sitting $(r=-0.15, P<0.01)$ and MVPA and television viewing $(r=-0.07, P<0.01)$.

Higher amounts of sedentary behavior were positively associated with all-cause mortality after adjustment for age, sex, education, smoking, diet, race, and MVPA (Table 2). Television viewing was positively associated with all-cause, cardiovascular, and cancer mortality and other causes of death after adjustment for age, sex, education, smoking, diet, and race (Table 2). Further adjustment for MVPA attenuated these associations only slightly. There was a graded elevation in risk that appeared to initially increase among adults reporting 3-4 h television watching/d. The greatest risk was noted for those watching the most television. Compared with those reporting $<1 \mathrm{~h}$ television viewing/d, participants reporting $\geq 7 \mathrm{~h}$ viewing/d had a $60 \%$ greater risk of all-cause mortality (HR: 1.61; 95\% CI: 1.47 , 1.76), nearly twice the risk of cardiovascular mortality (HR:

\section{TABLE 2}

Associations between television viewing, overall sitting, and mortality in 240,819 participants reporting no heart disease or cancer at baseline: NIH-AARP Diet and Health Study

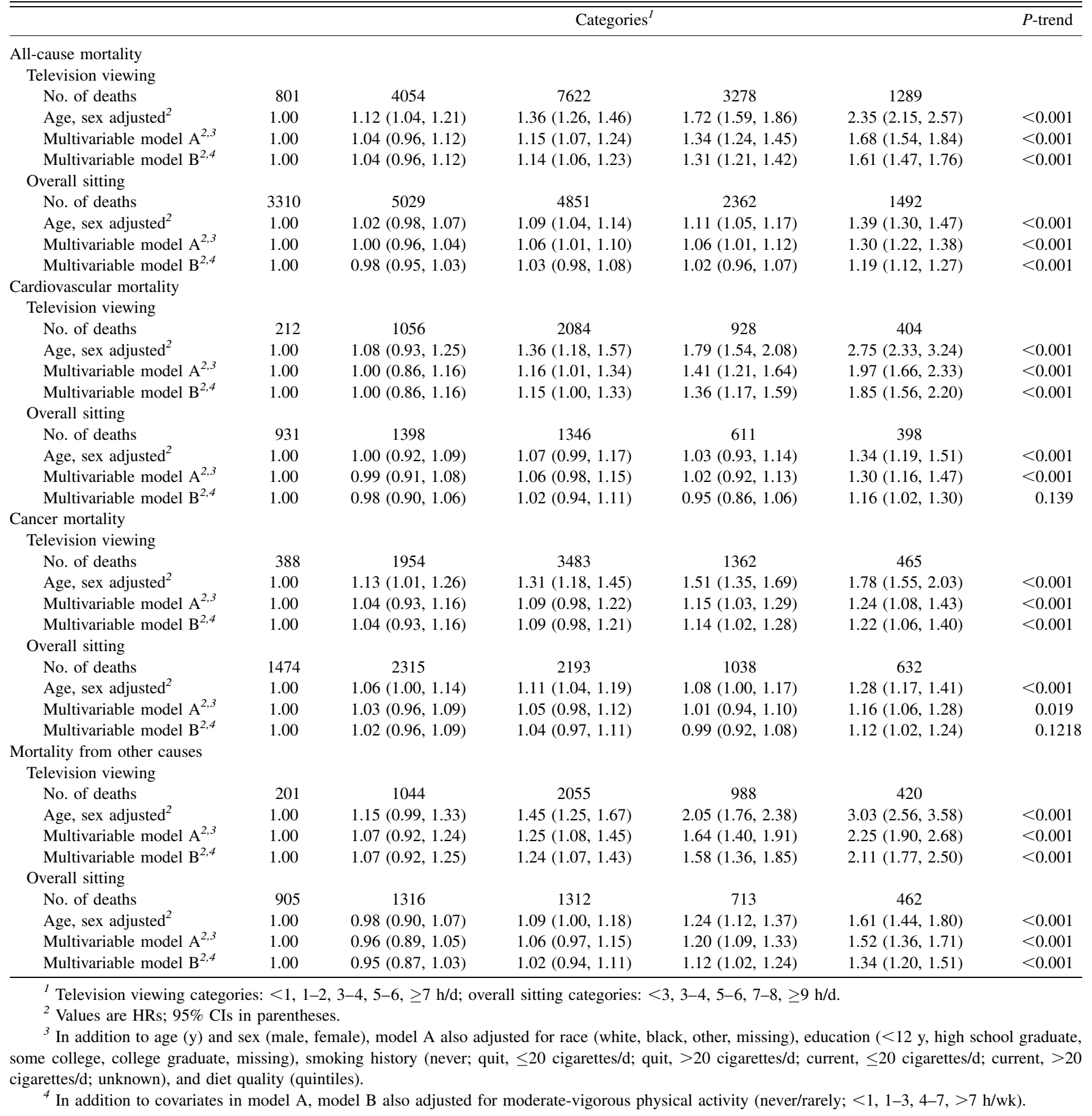


1.85; $95 \%$ CI: $1.56,2.20$ ), and a $20 \%$ greater risk of cancer mortality (HR: 1.22; 95\% CI: 1.06, 1.40) after adjustment for MVPA. Similar patterns of association were observed for overall sitting time, but the associations were weaker than for television viewing, and associations with cardiovascular and cancer mortality were no longer significant after adjustment for MVPA. Additional adjustment for BMI had only modest impact on results. After adjustment for BMI at study baseline, those reporting $\geq 7 \mathrm{~h}$ television viewing/d, remained at greater risk of all-cause (HR: $1.51 ; 95 \%$ CI: 1.38, 1.65), cardiovascular (HR: 1.62; 95\% CI: $1.37,1.93$ ), and cancer (HR: 1.18 ; 95\% CI: 1.03 , 1.36) mortality. Results for associations presented in Table 2, after additional adjustment for BMI, are presented in Supplementary Table 1 under "Supplemental data" in the online issue.

To examine the extent to which participation in MVPA was able to mitigate the increased risk of mortality related to high amounts of television viewing, we first evaluated the main effect of MVPA and then the joint effects of the 2 exposures by using a common low-risk referent group (ie, most active and least sedentary). We observed the expected strong inverse relation between MVPA and all-cause mortality in models adjusted for age, sex, education, smoking, diet, and race as follows- $\mathrm{MVPA}_{\text {Never/Rarely }}: 1.0$ (referent); $\mathrm{MVPA}_{<1 \mathrm{~h} / \mathrm{wk}}$ : HR, 0.87 (95\% CI: 0.77, 0.86); MVPA $_{1-3 \mathrm{~h} / \mathrm{wk}}$ : HR, $0.74(95 \%$ CI: 0.71, 0.76); $\mathrm{MVPA}_{4-7} \mathrm{~h} / \mathrm{wk}$ : HR, 0.69 (95\% CI: 0.66, 0.72); and MVPA $_{>7} \mathrm{~h} / \mathrm{wk}:$ HR, 0.61 (95\% CI: 0.58, 0.64). In joint-effects models presented in Figure 1, higher amounts of television viewing were associated with elevated risk of all-cause and cardiovascular mortality at both low and high levels of MVPA. Even among participants who reported levels of MVPA that approximated or exceeded currently recommended activity levels (ie, 1-3, 4-7, or $>7 \mathrm{~h}$ MVPA/wk), television viewing for $\geq 7 \mathrm{~h} / \mathrm{d}$ remained associated with a 1.5- to 2-fold increase in risk of all-cause mortality [MVPA $_{1-3} \mathrm{~h} / \mathrm{wk}$ : HR, 1.95 (95\% CI: 1.63, 2.35); MVPA $_{4-7} \mathrm{~h} / \mathrm{wk}$ :

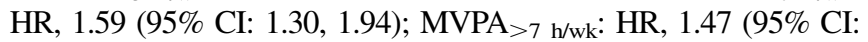
$1.20,1.79)]$ and an approximately 2 - to 2.5 -fold increase in risk of cardiovascular mortality $\left[\mathrm{MVPA}_{1-3} \mathrm{~h} / \mathrm{wk}\right.$ : HR, 2.63 (95\% CI: 1.81, 3.84); MVPA $_{4-7 \mathrm{~h} / \mathrm{wk}}:$ HR, 2.38 (95\% CI: 1.61, 3.53); $\mathrm{MVPA}_{>7 \mathrm{~h} / \mathrm{wk}}$ : HR, 2.00 (95\% CI: 1.33, 3.00); Figure 1].

In stratified analyses for television viewing and all-cause mortality (Table 3), we observed consistent positive associations in men and women, in all age-groups, in whites and blacks, and among participants with different levels of education, BMI, and smoking history and among those with diabetes, hypertension, and high cholesterol concentrations. In addition, the positive association was evident among participants with either low or high diet quality and among those who reported never smoking.

Finally, a sensitivity analysis was conducted to evaluate the potential for reverse causality to influence our primary results by excluding deaths occurring in the first 3 y of follow-up. We found no evidence of reverse causality. For example, after these exclusions, those reporting $\geq 7 \mathrm{~h}$ television viewing/d compared with $<1 \mathrm{~h} / \mathrm{d}$ remained at increased risk of all-cause (HR: 1.60; 95\% CI: 1.44 , 1.77) and cardiovascular (HR: 2.09; 95\% CI: 1.71, 2.55) mortality.

\section{DISCUSSION}

In this large prospective study in US adults, we found that prolonged television viewing and overall sitting time was positively associated with all-cause mortality after adjustment for MVPA and other potential risk factors. Higher amounts of television viewing were also positively associated with mortality from cardiovascular disease and cancer. Moreover, even among individuals reporting high levels of MVPA $(>7 \mathrm{~h} / \mathrm{wk})$, viewing television for $\geq 7 \mathrm{~h} / \mathrm{d}$ was associated with $50 \%$ greater risk of all-cause mortality and a 2-fold greater risk of cardiovascular mortality. Thus, achieving currently recommended levels of MVPA, or a minimum of $2.5 \mathrm{~h}$ of moderate or $1.25 \mathrm{~h}$ of vigorous activity/wk (22), may not fully protect against high amounts of sedentary behavior. These findings underscore the need to educate clinicians and the public about the adverse consequences of prolonged time in sedentary behavior, particularly sitting and watching television. In addition to regular participation in MVPA at recommended levels, adults also should reduce sedentary time when possible, in favor of more active pursuits.

To date, most research in adults on the adverse health effects of television viewing has focused on unhealthy dietary behaviors and obesity, with less attention given to its impact on physical activity levels. The impact of television viewing on physical activity levels, however, can be substantial. A recent randomized trial found that a $2.9-\mathrm{h} / \mathrm{d}$ reduction in television viewing resulted in a significant increase in physical activity of $\sim 120 \mathrm{kcal} / \mathrm{d}$ and a nonsignificant reduction in energy intake of $100 \mathrm{kcal} / \mathrm{d}(23)$, indicating that television viewing can influence physical activity as strongly as dietary behaviors. Interestingly, the increased physical activity resulting from reduced television viewing primarily came from light intensity activity. We believe that a displacement of time spent in lower intensity nonexercise activities by sedentary behavior is a logical behavioral mechanism explaining the associations observed in the present study.

Can a few hours of additional sitting really reduce overall physical activity levels enough to modify disease risk? We estimate that if $1 \mathrm{~h}$ of sedentary behavior, such as television viewing, results in the displacement of $1 \mathrm{~h}$ of light-intensity activity, the amount of physical activity energy expenditure lost would be $\sim 1$ metabolic equivalent task-hour/d (or $1 \mathrm{kcal} \cdot \mathrm{kg}^{-1} \cdot \mathrm{d}^{-1}$ ) (4), which is roughly equivalent to $15 \mathrm{~min}$ walking/d. Thus, several additional hours spent sedentary can quickly displace a substantial amount of physical activity (3). In this context, our results are consistent with those of Manini et al (24) and others (25) indicating that lower intensity nonexercise activities may provide considerable mortality benefit. Research is needed to understand more completely the optimal balance between time spent in active and sedentary behaviors and the health effects associated with lower intensity nonexercise activities (26).

Our findings confirm and extend recent etiologic studies examining the relation between sedentary behavior and mortality (10-12, 27). For example, Dunstan et al (12) found that adults who reported $\geq 4$ h of television viewing/d (compared with $<2$ $\mathrm{h} / \mathrm{d}$ ) had a $50 \%$ increase in risk of all-cause and nearly a 2-fold greater risk of cardiovascular mortality. Similar findings have been noted by other prospective studies $(11,27)$. Our finding for overall sitting and all-cause mortality was also consistent with similar studies conducted in Canadian adults (10) and Japanese men (28). Collectively, results from these studies provide consistent and strong observational support for the hypothesis that prolonged time spent in sedentary behaviors is independently associated with increased risk of early mortality.

Our most striking finding was that individuals who reported participating in $>7 \mathrm{~h}$ MVPA/wk, but who also watched $\geq 7 \mathrm{~h}$ television/d had a $50 \%$ greater risk of death from all causes and 
A

All-cause mortality

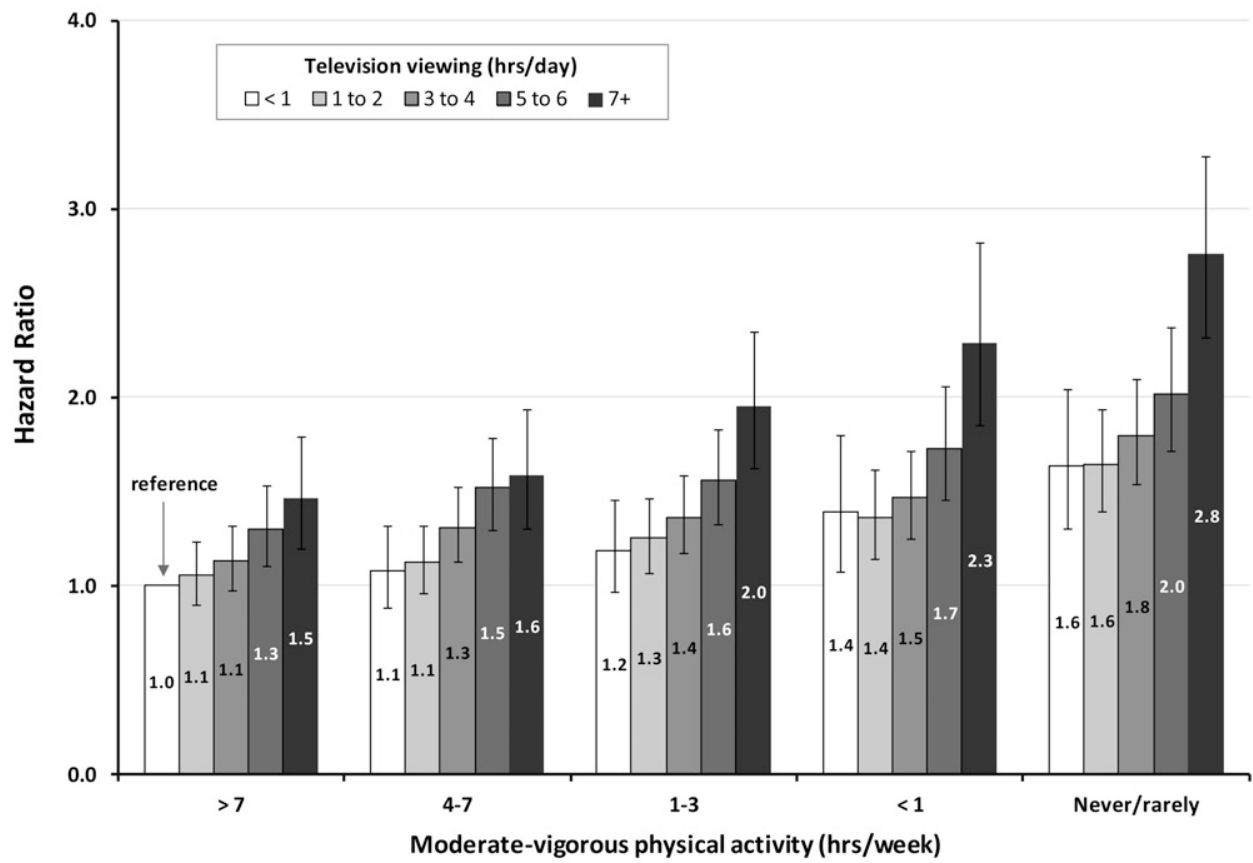

B

Cardiovascular mortality

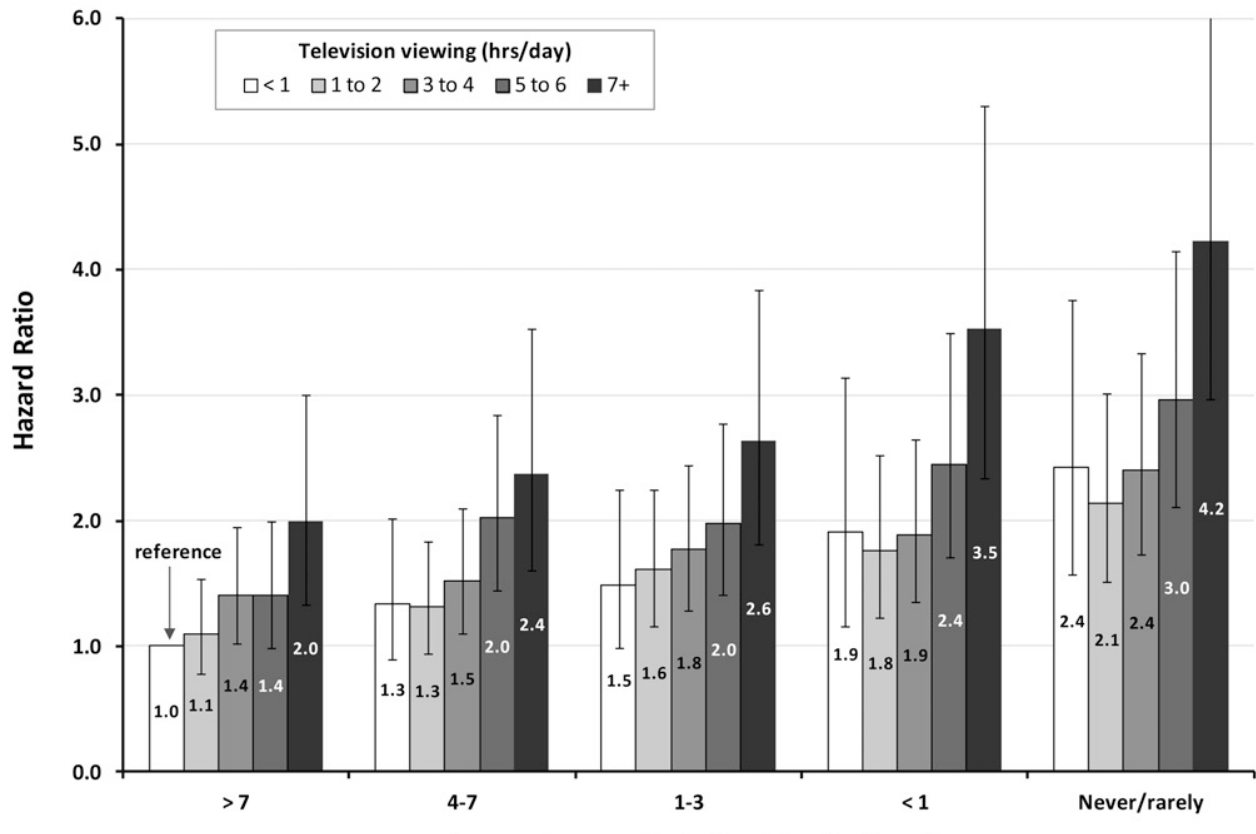

Moderate-vigorous physical activity (hrs/week)

FIGURE 1. Joint effects on mortality for MVPA (h/wk) and television viewing (h/d): NIH-AARP Diet and Health Study. Values are HRs and 95\% CIs. Values were adjusted for age (y), sex (male, female), race (white, black, other, missing), education ( $<12 \mathrm{y}$, high school graduate, some college, college graduate, missing), smoking history (never; quit, $\leq 20$ cigarettes/d; quit, $>20$ cigarettes/d; current, $\leq 20$ cigarettes/d; current, $>20$ cigarettes/d; unknown), and diet quality (quintiles). A: All-cause mortality. All tests for trend within each level of MVPA were significant $(P<0.001)$. The test for multiplicative interaction between MVPA and television viewing was significant $(P=0.002)$. B: Cardiovascular mortality. All tests for trend within each level of MVPA were significant $(P<0.001)$. The test for multiplicative interaction between MVPA and television viewing was not significant $(P=0.198)$. MVPA, moderatevigorous physical activity.

twice the risk of death from cardiovascular disease. Clearly, in these data, participation in a substantial amount of MVPA failed to provide protection for mortality risk in the face of a high amount of television watching, which is the most prevalent leisure-time sedentary behavior in the United States (29). These findings call into question whether MVPA alone is sufficient to control mortality risk in adults who spend large amounts of time engaged in sedentary behaviors. 
TABLE 3

Association between television viewing and all-cause mortality in 240,819 participants reporting no heart disease or cancer at baseline, by selected participant characteristics: NIH-AARP Diet and Health Study ${ }^{1}$

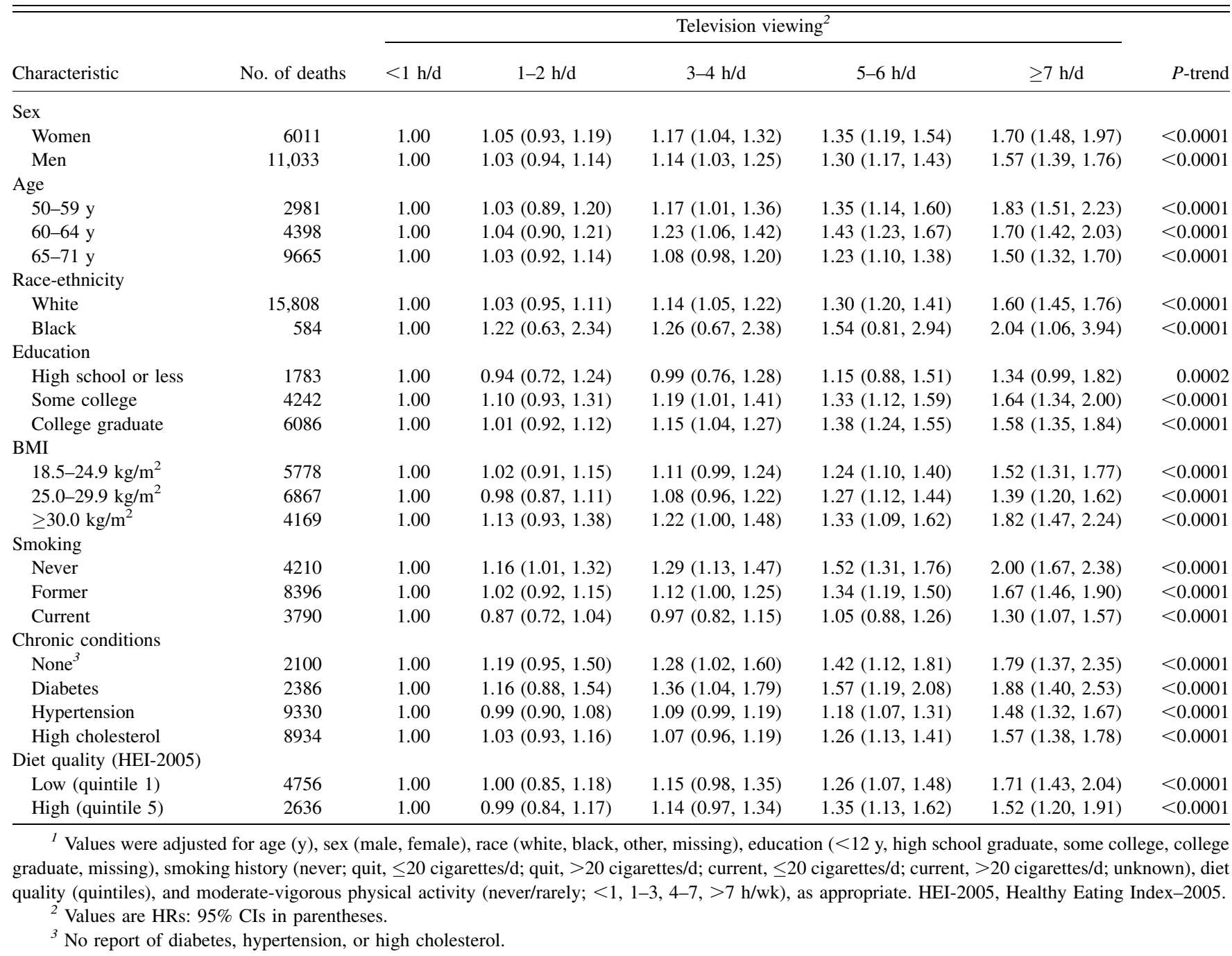

A unique feature of this study was our assessment of both overall sitting time and television viewing, and the stronger mortality associations we observed for television viewing merits comment. At least 2 plausible explanations could explain this result. First, television viewing could be more deleterious than other kinds of sedentary behavior. However, this seems unlikely because mortality rates are higher among those with sedentary occupations compared with those with active occupations (eg, 30, 31), and cardiovascular mortality is higher among those who spend high amounts of time in car travel compared with those who spend low amounts of time in car travel (32), suggesting that a broad range of sedentary behaviors may affect health adversely. A more likely explanation is that the measurement properties of the 2 exposures vary significantly. Compared with specific questions about individual sedentary behaviors, such as television viewing, single questions that assess overall sitting time are more cognitively challenging (33) and often have lower validity (34). Higher levels of measurement error for the overall sitting time question in most cases would result in attenuation in the strength of the observable risk estimates (35). Better measures of overall sitting time in prospective studies (36) will be required to estimate more accurately the risks associated with this omnipresent exposure.
Strengths of our study include its large sample size, prospective design, and ability to carefully evaluate the potential influence of confounding and reverse causality on our results. Results from sensitivity analyses (excluding early deaths) and our finding of a positive association between sedentary behaviors and mortality among adults who reported participation in substantial levels of MVPA argues against the notion that reverse causality explained these findings. Our study also had a number of limitations that should be considered. We used self-reports of sedentary behavior and MVPA. Whereas there is evidence for acceptable reliability and validity for our approach to assessing these behaviors (16), measurement error may nonetheless have attenuated the associations observed. Residual confounding is also a concern. However, although we cannot rule out this potential bias completely, our stratified analyses showed positive associations between television viewing and mortality in adults who were younger, more educated, never smokers, of normal weight, and who reported healthier diets. These findings argue against residual confounding as a major bias in the present study. In addition, although we completed a detailed examination of many potential confounders, we did not have information about 
occupational status at study baseline. Last, our results are most applicable to adults in the age range of our study population.

In conclusion, sedentary behaviors are ubiquitous in modern life, and sitting at home in front of the television, traveling in automobiles, and sitting in the workplace are among the most prevalent sedentary behaviors $(29,37)$. Our finding that participation in $>7 \mathrm{~h}$ of MVPA/wk failed to fully mitigate mortality risks associated with prolonged television viewing adds to the evidence base indicating that both sedentary behaviors and MVPA are associated with mortality and emphasizes the need to address both of these health behaviors in future intervention efforts. Strong public health messages that encourage reductions in sedentary time are an important additional lever that has yet to be forcefully pulled in the effort to increase overall physical activity levels in the population and to reduce disease risk. Adults should be encouraged to reduce time spent in sedentary behaviors, when possible, in favor of more active pursuits and to participate in MVPA at recommended levels.

We are indebted to the participants in the NIH-AARP Diet and Health Study for their outstanding cooperation.

The authors' responsibilities were as follows-CEM: had full access to all of the data in the study and takes responsibility for the integrity of the data and the accuracy of the data analysis; CEM, SMG, SCM, and AS: study concept and design; AH and AS: acquisition of data; CEM, SMG, SCM, $\mathrm{HRB}, \mathrm{AB}, \mathrm{YP}, \mathrm{RPT}$, and AS: analysis and interpretation of data; CEM, SMG, SCM, HRB, and RPT: drafting of the manuscript; CEM, YP, AB, $\mathrm{AH}$, and AS: critical revision of the manuscript for important intellectual content; CEM, SMG, and SCM: statistical analysis; YP, AH, and AS: administrative, technical, and material support; and AS: obtained funding and study supervision. None of the authors reported a conflict of interest.

\section{REFERENCES}

1. Brownson RC, Boehmer TK, Luke DA, Brownson RC, Boehmer TK, Luke DA. Declining rates of physical activity in the United States: what are the contributors? Annu Rev Public Health 2005;26:421-43.

2. Pate RR, O'Neill J, Lobelo F. The evolving definition of "sedentary". Exerc Sport Sci Rev 2008;36:173-8.

3. Owen N, Sparling PB, Healy GvN, Dunstan DW, Matthews CE. Sedentary behavior: emerging evidence for a new health risk. Mayo Clin Proc 2010;85:1138-41.

4. Matthews CE, Chen KY, Freedson PS, Buchowski MS, Beech BM, Pate RR, Troiano RP. Amount of time spent in sedentary behaviors: United States 2003-2004. Am J Epidemiol 2008;167:875-81.

5. Levine JA, Lanningham-Foster LM, McCrady SK, Krizan AC, Olson LR, Kane PH, Jensen MD, Clark MM. Interindividual variation in posture allocation: possible role in human obesity. Science 2005;307: $584-6$.

6. Hu FB, Li TY, Colditz GA, Willett WC, Manson JE. Television watching and other sedentary behaviors in relation to risk of obesity and type 2 diabetes mellitus in women. JAMA 2003;289:1785-91.

7. Dunstan DW, Salmon J, Healy GN, Shaw JE, Jolley D, Zimmet PZ, Owen N, on behalf of the AusDiab Steering Committee. Association of television viewing with fasting and 2-h postchallenge plasma glucose levels in adults without diagnosed diabetes. Diabetes Care 2007;30: 516-22.

8. Healy GN, Matthews CE, Dunstan DW, Winkler EAH, Owen N. Sedentary time and cardio-metabolic biomarkers in US adults: NHANES 2003-2006. Eur Heart J 2011;32:590-7.

9. Healy GN, Dunstan DW, Salmon J, Cerin E, Shaw JE, Zimmet PZ, Owen N. Objectively measured light-intensity physical activity is independently associated with 2-h plasma glucose. Diabetes Care 2007;30: 1384-9.

10. Katzmarzyk PT, Church TS, Craig CL, Bouchard C. Sitting time and mortality from all causes, cardiovascular disease, and cancer. Med Sci Sports Exerc 2009;41:998-1005.

11. Patel AV, Bernstein L, Deka A, Feigelson HS, Campbell PT, Gapstur SM, Colditz GA, Thun MJ. Leisure time spent sitting in relation to total mortality in a prospective cohort of US adults. Am J Epidemiol 2010;172:419-29.

12. Dunstan DW, Barr ELM, Healy GN, Salmon J, Shaw JE, Balkau B, Magliano DJ, Cameron AJ, Zimmet PZ, Owen N. Television viewing time and mortality: the Australian Diabetes, Obesity and Lifestyle Study (AusDiab). Circulation 2010;121:384-91.

13. Schatzkin A, Subar AF, Thompson FE, Harlan LC, Tangrea J, Hollenbeck AR, Hurwitz PE, Coyle L, Schussler N, Michaud DS, et al. Design and serendipity in establishing a large cohort with wide dietary intake distributions: the National Institutes of Health-American Association of Retired Persons Diet and Health Study. Am J Epidemiol 2001;154:1119-25.

14. Hill ME, Rosenwaike I. The Social Security Administration's Death Master File: the completeness of death reporting at older ages. Soc Secur Bull 2001;64:45-51.

15. Rich-Edwards JW, Corsano KA, Stampfer MJ. Test of the National Death Index and Equifax Nationwide Death Search. Am J Epidemiol 1994;140:1016-9.

16. Clark BK, Sugiyama T, Healy GN, Salmon J, Dunstan DW, Owen N. Validity and reliability of measures of television viewing time and other non-occupational sedentary behavior in adults: a review. Obes Rev 2009;10:7-16.

17. Washburn RA, Smith KW, Jette AM, Janney CA. The Physical Activity Scale for the Elderly (PASE): development and evaluation. J Clin Epidemiol 1993;46:153-62.

18. Leitzmann MF, Park Y, Blair A, Ballard-Barbash R, Mouw T, Hollenbeck AR, Schatzkin A. Physical activity recommendations and decreased risk of mortality. Arch Intern Med 2007;167:2453-60.

19. Guenther PM, Reedy J, Krebs-Smith SM. Development of the Healthy Eating Index-2005. J Am Diet Assoc 2008;108:1896-901.

20. Levine JA, Eberhardt NL, Jensen MD. Role of nonexercise activity thermogenesis in resistance to fat gain in humans. Science 1999;283:212-4.

21. Rothman KJ, Greenland S. Modern epidemiology. Philadelphia, PA: Lippincott-Raven, 1998.

22. Physical Activity Guidelines Advisory Committee. Physical Activity Guidelines Advisory Committee Report. Washington, DC: US Department of Health and Human Services, 2008.

23. Otten JJ, Jones KE, Littenberg B, Harvey-Berino J. Effects of television viewing reduction on energy intake and expenditure in overweight and obese adults: a randomized controlled trial. Arch Intern Med 2009; 169:2109-15.

24. Manini TM, Everhart JE, Patel KV, Schoeller DA, Colbert LH, Visser M, Tylavsky F, Bauer DC, Goodpaster BH, Harris TB. Daily activity energy expenditure and mortality among older adults. JAMA 2006;296: $171-9$.

25. Matthews CE, Jurj AL, Shu XO, Li H, Yang G, Gao Y-T, Zheng W. Influence of exercise, walking, cycling, and overall non-exercise physical activity on mortality in Chinese women. Am J Epidemiol 2007; 165:1343-50.

26. Powell KE, Paluch AE, Blair SN. Physical activity for health: what kind? How much? How intense? On top of what? Annu Rev Public Health 2011;32:349-65.

27. Wijndaele K, Brage S, Besson H, Khaw KT, Sharp SJ, Luben R, Wareham NJ, Ekelund U. Television viewing time independently predicts all-cause and cardiovascular mortality: the EPIC Norfolk Study. Int J Epidemiol 2011;40:150-9.

28. Inoue M, Iso H, Yamamoto S, Kurahashi N, Iwasaki M, Sasazuki S, Tsugane S. Daily total physical activity level and premature death in men and women: results from a large-scale population-based cohort study in Japan (JPHC Study). Ann Epidemiol 2008;18:522-30.

29. American Time Use Survey, Bureau of Labor Statistics. Time spent in primary activities in the American Time Use Survey (Table A-1) 2009. Available from: http://www.bls.gov/tus/tables/a1_2009.pdf (accessed 7 October 2010).

30. Barengo NC, Hu G, Lakka TA, Pekkarinen H, Nissinen A, Tuomilehto J. Low physical activity as a predictor for total and cardiovascular disease mortality in middle-aged men and women in Finland. Eur Heart J 2004; 25:2204-11.

31. Andersen LB, Schnohr P, Schroll M, Hein HO. All-cause mortality associated with physical activity during leisure time, work, sports, and cycling to work. Arch Intern Med 2000;160:1621-8.

32. Warren TY, Barry V, Hooker SP, Sui X, Church TS, Blair SN. Sedentary behaviors increse risk of cardiovascular disease mortality in men. Med Sci Sports Exerc 2010;42:879-85. 
33. van Uffelen JG, Heesch K, Hill R, Brown W. A qualitative study of older adults' responses to sitting-time questions: do we get the information we want? BMC Public Health 2011;11:458.

34. Healy GN, Clark B, Winkler EAH, Gardiner PA, Brown WJ, Matthews CE. Measurement of Adults' Sedentary Time in Population-Based Studies. Am J Prev Med 2011;41:216-27.

35. Ferrari P, Friedenreich C, Matthews CE. The role of measurement error in estimating levels of physical activity. Am J Epidemiol 2007;166:832-40.
36. Schatzkin A, Subar AF, Moore S, Park Y, Potischman N, Thompson FE, Leitzmann M, Hollenbeck A, Morrissey KG, Kipnis V. Observational epidemiologic studies of nutrition and cancer: the next generation (with better observation). Cancer Epidemiol Biomarkers Prev 2009;18: 1026-32.

37. Tudor-Locke C, Ainsworth BE, Washington TL, Troiano RP. Assigning metabolic equivalent (MET) values to the 2002 Census Occupational Classification System. J Phys Act Health 2011;8:581-6. 